\title{
Complications from Plaque versus Proton Beam Therapy for Choroidal Melanoma: A Qualitative Systematic Review
}

\author{
Victoria L. Tseng1,2, Anne L. Coleman", ${ }^{1,2}$ Zuo-Feng Zhang'2, Tara A. McCannel1 \\ ${ }^{1}$ Stein Eye Institute, David Geffen School of Medicine at UCLA, Los Angeles, CA, USA \\ ${ }^{2}$ Department of Epidemiology, UCLA Fielding School of Public Health, Los Angeles, CA, USA \\ Email: young@jsei.ucla.edu
}

Received 24 December 2015; accepted 6 March 2016; published 10 March 2016

Copyright $@ 2016$ by authors and Scientific Research Publishing Inc.

This work is licensed under the Creative Commons Attribution International License (CC BY). http://creativecommons.org/licenses/by/4.0/

\section{(c) (i) Open Access}

\section{Abstract}

Plaque brachytherapy has been a mainstay of treatment for choroidal melanoma to achieve intraocular tumor control. The most common radioisotopes used for treating smaller sized tumors are Iodine-125 in North America and Ruthenium-106 in Europe. Proton beam radiotherapy is available at a few centers and may also be used to achieve local tumor control. Both plaque and proton beam therapy are known to be associated with a range of complications that may affect visual outcome and quality of life. These include radiation retinopathy, optic neuropathy, neovascular glaucoma and local treatment failure, requiring enucleation. While differences in the rates of these complications have not been well established in the literature for patients treated with plaque versus proton beam therapy for choroidal melanoma, certain geographic regions prefer one treatment modality over the other. The purpose of this qualitative systematic review was to compare and contrast reported complications that developed with plaque and proton beam therapy for the treatment of choroidal melanoma in studies published over a ten-year period. Reported rates suggest that patients with proton beam therapy had potentially higher rates of complications, including vision loss, enucleation, and neovascular glaucoma compared to those with plaque therapy. The rates of optic neuropathy, radiation retinopathy, and cataract formation were widely variable for the two treatment modalities and rates of metastasis and metastasis-free survival appeared similar with both treatments. The most common reported predictors of ocular complications following both types of therapy were tumor distance from the optic nerve, tumor thickness, and radiation dose, suggesting that inherent tumor characteristics play a role in visual prognosis. 


\section{Keywords}

\section{Choroidal Melanoma, Uveal Melanoma, Plaque Therapy, Brachytherapy, Proton Therapy, Treatment Complications, Metastasis, Enucleation}

\section{Introduction}

Choroidal melanoma, also known as posterior uveal melanoma, arises from melanocytes within the choroid. It is the most common primary intraocular tumor of adults and the second most common type of malignant melanoma after cutaneous melanoma. Despite advances in its diagnosis and treatment, the incidence of choroidal melanoma in the United States has remained unchanged since the 1970s, with a slight male predominance and peak incidence in the fifth to sixth decades of life [1]-[3]. Thirty to fifty percent of patients succumb to metastatic disease within ten years of diagnosis, with worse prognosis associated with the clinical factors of older age, greater tumor size, and ciliary body location; the histological factors of epithelioid cellular morphology and presence of vascular loops; and with the molecular finding of loss of chromosome 3 within the tumor tissue [4] [5].

In 2001, the Collaborative Ocular Melanoma Study (COMS) reported no difference in mortality 12 years following treatment with iodine-125 brachytherapy versus enucleation for medium sized tumors [6]. Since then, brachytherapy has been a globe-sparing treatment of choice, while enucleation may be recommended for large tumors. Proton beam radiotherapy is another globe-sparing treatment alternative for both medium and large choroidal melanoma [7].

Both plaque and proton beam therapy are known to be associated with a range of ocular complications including cataract formation, radiation retinopathy, optic neuropathy and neovascular glaucoma; however, the differences in the side effect profiles of the two treatment modalities have not been well explored [8] [9]. Additionally, varying rates of distant metastasis have been reported with the two treatments without an obvious benefit of one treatment over the other for long-term survival [10]-[26]. However, there are some geographic treatment pattern preferences for one treatment modality over the other despite a lack of convincing data in the published literature. To this end, the purpose of this review was to compare ocular complications for patients receiving plaque and proton beam radiotherapy for choroidal melanoma.

\section{Search Strategy}

A literature search was performed for this qualitative systematic review [27] using the following keywords on PubMed [28]: "side effects AND choroidal melanoma AND plaque therapy”, "visual outcomes AND choroidal melanoma AND plaque therapy”, "visual acuity AND choroidal melanoma AND plaque therapy”, "eye preservation AND choroidal melanoma AND plaque therapy”, "optic neuropathy AND choroidal melanoma AND plaque therapy”, "retinopathy AND choroidal melanoma AND plaque therapy”, "neovascular glaucoma AND choroidal melanoma AND plaque therapy”, “cataract AND choroidal melanoma AND plaque therapy”, and "metastasis AND choroidal melanoma AND plaque therapy". For studies examining complications from proton beam therapy, we used the same search terms but replaced "plaque therapy" with "proton beam therapy" in each search term. Our inclusion criteria were 1) study published in the English language, 2) examination of at least a subset of patients treated initially with plaque therapy or proton beam therapy without initial adjunct treatments, and 3) examination of at least one of visual acuity, eye preservation, optic neuropathy, radiation retinopathy, neovascular glaucoma, cataract, and distant metastasis as an outcome following treatment for choroidal melanoma, and 4) publication over a ten-year period between January 1, 2005 and December 31, 2014. These inclusion criteria were selected based on the desire to examine ocular complications directly related to plaque or proton beam therapy in a recent time period when both treatment modalities were used frequently.

From each study, the following information was extracted: sample size, tumor type, duration of follow-up, rates of each outcome (visual acuity worse than 20/200, enucleation, optic neuropathy, radiation retinopathy, neovascular glaucoma, cataract, and distant metastasis), and baseline predictors of each outcome. Due to significant heterogeneity across studies with regard to tumor characteristics, radioisotope used, duration of follow-up, and study population, weighted averages of each complication were not calculated. 


\section{Results}

\subsection{Overview of Included Studies}

The search strategy yielded 556 studies between 2005 and 2014, of which 29 were included in this review with the earliest published in 2005 and the latest in 2010. These included 22 studies of plaque therapy and 7 studies of proton beam therapy for choroidal melanoma. For studies of plaque therapy, sample sizes ranged from 24 to 650 patients and duration of follow-up ranged from an average of 23 to 60 months. For studies of proton beam therapy, sample sizes ranged from 18 to 349 patients and duration of follow-up ranged from an average of 28 to 78 months.

\subsection{Visual Acuity}

Studies examining visual acuity following plaque therapy for choroidal melanoma are outlined in Table 1 and Table 2. Visual acuity can be temporarily or permanently compromised following treatment for choroidal melanoma for a variety of reasons including radiation-induced damage to the anterior segment, retina, and optic nerve. Of the 15 included studies [10]-[20] [29]-[31] that examined visual acuity as an outcome following plaque therapy, sample sizes ranged from 24 to 650 patients and follow-up ranged from a mean of 23 months to five years. In five of six studies that examined the proportion of patients with visual acuity better than 20/40, less than $50 \%$ of patients were able to preserve visual acuity equal to or better than 20/40 at last follow-up. Fourteen studies examined the proportion of patients with visual acuity equal to or better than 20/200 with a wide range of proportions reported ( $23 \%$ - 94.4\%). Factors shown to be associated with poor long term visual acuity following plaque therapy in multivariate models included radiation dose and tumor location [13] [30] [32], with two studies [13] [32] demonstrating that tumor location near the optic nerve predicted poor long term visual function.

There were seven studies [9] [23]-[26] [34] included in this review that examined visual acuity as an outcome following proton beam therapy. The sample size in these studies ranged from 18 to 147 patients, and follow-up ranged from a median of 28 to 78 months. Compared to studies of plaque therapy, there were more studies that focused on large tumors and tumors close to the optic nerve. Most studies did not examine preservation of visual acuity equal to or better than $20 / 40$ as an outcome, with only one study [23] reporting that $44.8 \%$ of patients had visual acuity equal to or better than 20/40 at five years following the initiation of treatment. Presumably, most studies only focused on preservation of visual acuity equal to or better than 20/200 as a long term outcome because proton beam therapy was associated with more visual loss compared to plaque therapy; the majority of studies [9] [24] [25] [33] [34] included in this review found that less than $50 \%$ of patients were able to preserve visual acuity equal or greater than 20/200 at the end of follow-up after proton beam therapy. The only study that examined predictors of poor visual acuity following proton beam therapy [27] found that initial degree of ocular involvement, tumor height, and initial visual acuity were predictive of poor visual acuity following therapy.

\subsection{Eye Preservation}

Studies examining eye preservation following plaque and proton beam therapy for choroidal melanoma are outlined in Table 3 and Table 4. Secondary enucleation for choroidal melanoma is most often performed for tumor recurrence or treatment toxicity [33]. While enucleation may achieve local tumor control and provide symptomatic relief, it may also be associated with cosmetic side effects and potential detriment to quality of life [34]. There were 13 studies [10]-[14] [16]-[18] [20] [29]-[32] included in this review that examined eye preservation following plaque therapy for choroidal melanoma. Sample sizes ranged from 24 to 650 patients and mean follow-up ranged from 23 months to five years. The majority of studies of plaque therapy reported less than $10 \%$ enucleation rates for tumor recurrence [10] [11] [13] [17] [18] [20] [31], while enucleation rates for treatment toxicity ranged from $1.6 \%$ [13] to $14.2 \%$ [11]. The only study that examined predictors of enucleation [32] following plaque therapy found that plaque size, tumor recurrence, neovascular glaucoma, and nonproliferative retinopathy were predictive of requiring enucleation.

There were seven included studies [9] [23]-[26] [34] [35] that examined eye preservation following proton beam therapy for choroidal melanoma. Sample sizes ranged from 21 to 349 patients and follow-up time ranged from a mean of 28 months to a median of 78 months. While most studies reported similar secondary enucleation rates when compared to studies of plaque therapy, three studies [9] [24] [35] reported cumulative enucleation 
Table 1. Visual acuity outcomes following plaque therapy for choroidal melanoma.

\begin{tabular}{|c|c|c|c|c|c|c|c|}
\hline Study & $\begin{array}{l}\text { Sample } \\
\text { Size }\end{array}$ & Tumor Type & $\begin{array}{l}\text { Duration of } \\
\text { Follow-Up }\end{array}$ & $\begin{array}{c}\text { Number (\%) } \\
\text { with VA } \\
\geq 20 / 40 \text { at } \\
\text { End of } \\
\text { Follow-Up }\end{array}$ & $\begin{array}{l}\text { Number (\%) } \\
\text { with VA } \\
\geq 20 / 200 \text { at } \\
\text { End of } \\
\text { Follow-Up }\end{array}$ & $\begin{array}{l}\text { Alternate } \\
\text { Measure of } \\
\text { VA }\end{array}$ & $\begin{array}{l}\text { Predictors of } \\
\text { Poor VA after } \\
\text { Treatment }\end{array}$ \\
\hline $\begin{array}{l}\text { Bergman et al. } \\
2005 \text { [29] }\end{array}$ & 579 eyes & $\begin{array}{c}\text { Choroidal and ciliary } \\
\text { body melanomas }\end{array}$ & $\begin{array}{l}3 \text { and } 5 \\
\text { years }\end{array}$ & $\begin{array}{c}64 / 171 \\
(37.2 \%)\end{array}$ & $\begin{array}{c}67 / 171 \\
(39.2 \%)\end{array}$ & -- & -- \\
\hline $\begin{array}{l}\text { Sagoo et al. } \\
2007[10]\end{array}$ & 37 patients & $\begin{array}{c}\text { Circumpapillary } \\
\text { choroidal melanoma }\end{array}$ & $\begin{array}{l}\text { Mean } 52 \\
\text { months, } \\
\text { median } 46 \\
\text { months }\end{array}$ & -- & $\begin{array}{c}38 \% \\
\text { non-enucleated } \\
\text { eyes }\end{array}$ & -- & -- \\
\hline $\begin{array}{l}\text { Sagoo et al. } \\
2008 \text { [11] }\end{array}$ & 141 patients & $\begin{array}{c}\text { Juxtapapillary choroidal } \\
\text { melanoma overhanging } \\
\text { optic disc }\end{array}$ & $\begin{array}{l}\text { Mean } 56 \\
\text { months }\end{array}$ & -- & $23 \%$ & -- & -- \\
\hline $\begin{array}{l}\text { Finger et al. } \\
2010[30]\end{array}$ & 384 patients & $\begin{array}{l}\text { Uveal melanomas } \\
\text { treated with } \\
\text { Palladium-103 }\end{array}$ & $\begin{array}{l}\text { Mean } 47.2 \\
\text { months }\end{array}$ & -- & -- & $\begin{array}{l}\text { Low and medium } \\
\text { foveal doses: } \\
\text { End median VA } \\
\text { 20/25-20/30; High } \\
\text { foveal dose: End } \\
\text { median VA 20/80 }\end{array}$ & $\begin{array}{l}\text { Foveal dose } \\
\text { of radiation }\end{array}$ \\
\hline $\begin{array}{l}\text { Gunduz et al. } \\
2010 \text { [12] }\end{array}$ & 24 patients & $\begin{array}{l}\text { Choroidal melanoma } \\
\text { treated with ruthenium } \\
\text { brachytherapy alone }\end{array}$ & -- & $\begin{array}{c}3 / 21 \\
(14.2 \%)\end{array}$ & $\begin{array}{c}10 / 21 \\
(47.6 \%)\end{array}$ & -- & -- \\
\hline $\begin{array}{l}\text { Verschueren } \\
\text { et al. } 2010 \\
\text { [13] }\end{array}$ & 430 patients & $\begin{array}{l}\text { Choroidal } \\
\text { melanoma }\end{array}$ & $\begin{array}{l}\text { Median } 50 \\
\text { months }\end{array}$ & -- & $\begin{array}{l}283 / 430 \\
(65.8 \%)\end{array}$ & -- & $\begin{array}{l}\text { Central tumors, } \\
\text { juxtapapillary } \\
\text { tumors }\end{array}$ \\
\hline $\begin{array}{c}\text { Karlovits et al. } \\
2011[14]\end{array}$ & 35 patients & $\begin{array}{l}\text { Choroidal melanoma } \\
\text { treated with iodine } \\
\text { brachytherapy }\end{array}$ & $\begin{array}{l}\text { Median } 45 \\
\text { months }\end{array}$ & -- & $\begin{array}{l}32 \% \text { of patients } \\
\text { with } 3 \text { or more } \\
\text { years of } \\
\text { follow-up }\end{array}$ & -- & -- \\
\hline $\begin{array}{l}\text { Newman et al. } \\
2011[15]\end{array}$ & 50 patients & $\begin{array}{l}\text { Subfoveal choroidal } \\
\text { melanomas with } \geq 6 \\
\text { months of follow-up }\end{array}$ & $\begin{array}{l}\text { Median } 54 \\
\text { months }\end{array}$ & $\begin{array}{c}18 / 50 \\
(36.0 \%)\end{array}$ & $\begin{array}{c}25 / 50 \\
(50.0 \%)\end{array}$ & -- & -- \\
\hline $\begin{array}{l}\text { Finger et al. } \\
2012[16]\end{array}$ & 24 patients & $\begin{array}{c}\text { Choroidal melanomas } \\
\text { within } 1.5 \text { mm of optic } \\
\text { disc treated with slotted } \\
\text { radiotherapy }\end{array}$ & $\begin{array}{c}\text { Mean } 23 \\
\text { months }\end{array}$ & $\begin{array}{l}12 / 22 \\
(55 \%)\end{array}$ & $\begin{array}{l}17 / 22 \\
(77 \%)\end{array}$ & -- & -- \\
\hline $\begin{array}{l}\text { Berry et al. } \\
2013 \text { [17] }\end{array}$ & 82 patients & $\begin{array}{l}\text { Medium sized } \\
\text { choroidal melanomas }\end{array}$ & $\begin{array}{l}\text { Median } 46.8 \\
\text { months }\end{array}$ & $\begin{array}{c}29 / 82 \\
(35.4 \%)\end{array}$ & $\begin{array}{c}35 / 82 \\
(42.7 \%)\end{array}$ & -- & -- \\
\hline $\begin{array}{l}\text { Caminal et al. } \\
2013 \text { [18] }\end{array}$ & 54 patients & $\begin{array}{l}\text { Choroidal melanoma } \\
\text { treated with iodine } \\
\text { brachytherapy }\end{array}$ & $\begin{array}{l}\text { Mean } 59.47 \\
\text { months }\end{array}$ & -- & $\begin{array}{c}19 / 54 \\
(35.2 \%)\end{array}$ & -- & -- \\
\hline $\begin{array}{l}\text { Marconi et al. } \\
2013 \text { [31] }\end{array}$ & 94 patients & $\begin{array}{l}\text { Choroidal melanoma } \\
\text { treated with ruthenium } \\
\text { brachytherapy }\end{array}$ & $\begin{array}{l}\text { Median } 39 \\
\text { months }\end{array}$ & $30 \%$ & $56 \%$ & -- & -- \\
\hline $\begin{array}{c}\text { Semenova et al. } \\
2013 \text { [19] }\end{array}$ & 72 patients & $\begin{array}{l}\text { Small choroidal } \\
\text { melanomas }\end{array}$ & $\begin{array}{l}\text { Mean } 54 \\
\text { months }\end{array}$ & -- & $\begin{array}{c}68 / 72 \\
(94.4 \%)\end{array}$ & -- & -- \\
\hline $\begin{array}{l}\text { Sagoo et al. } \\
2014 \text { [32] }\end{array}$ & 650 eyes & $\begin{array}{c}\text { Juxtapapillary } \\
\text { choroidal melanoma }\end{array}$ & $\begin{array}{l}\text { Median } 40 \\
\text { months }\end{array}$ & - & $\begin{array}{l}46 \% \text { at } 5 \text { years, } \\
13 \% \text { at } 10 \text { years }\end{array}$ & -- & $\begin{array}{l}\text { Papillopathy, } \\
\text { radiation } \\
\text { cataract }\end{array}$ \\
\hline $\begin{array}{l}\text { Semenova et al. } \\
2014 \text { [20] }\end{array}$ & · 47 patients & $\begin{array}{l}\mathrm{T} 3 \text { or } \mathrm{T} 4 \text { sized choroidal } \\
\text { melanomas treated with } \\
\text { palladium brachytherapy }\end{array}$ & $\begin{array}{l}\text { Median } 47 \\
\text { months }\end{array}$ & & $\begin{array}{l}25 / 47 \\
(53 \%)\end{array}$ & -- & -- \\
\hline
\end{tabular}


Table 2. Visual acuity outcomes following proton beam therapy for choroidal melanoma.

\begin{tabular}{|c|c|c|c|c|c|c|c|}
\hline Study & Sample Size & Tumor Type & $\begin{array}{l}\text { Duration of } \\
\text { Follow-Up }\end{array}$ & $\begin{array}{l}\text { Number (\%) } \\
\text { with VA } \\
\geq 20 / 40 \text { at } \\
\text { End of } \\
\text { Follow-Up }\end{array}$ & $\begin{array}{l}\text { Number (\%) } \\
\text { with VA } \\
\geq 20 / 200 \text { at } \\
\text { End of } \\
\text { Follow-Up }\end{array}$ & $\begin{array}{c}\text { Alternate } \\
\text { Measure } \\
\text { of VA }\end{array}$ & $\begin{array}{l}\text { Predictors of Poor } \\
\text { VA after Treatment }\end{array}$ \\
\hline $\begin{array}{l}\text { Damato et al. } \\
2005 \text { [23] }\end{array}$ & 349 patients & Choroidal melanoma & $\begin{array}{l}\text { Median } 3.1 \\
\text { years }\end{array}$ & $\begin{array}{l}44.8 \% \text { at } \\
5 \text { years }\end{array}$ & $\begin{array}{l}61.1 \% \text { at } \\
5 \text { years }\end{array}$ & -- & $\begin{array}{l}\text { Ciliary body } \\
\text { involvement, basal } \\
\text { tumor dimension, retinal } \\
\text { invasion, extraocular } \\
\text { extension, tumor height, } \\
\text { reduced initial VA }\end{array}$ \\
\hline $\begin{array}{c}\text { Conway et al. } \\
2006 \text { [9] }\end{array}$ & 21 patients & $\begin{array}{c}\text { Extra large choroidal } \\
\text { or ciliochoroidal } \\
\text { melanomas }\end{array}$ & $\begin{array}{l}\text { Median } 28 \\
\text { months }\end{array}$ & -- & $\begin{array}{l}25 \% \text { after } 24 \\
\text { months }\end{array}$ & -- & -- \\
\hline $\begin{array}{l}\text { Kim et al. } \\
2010 \text { [33] }\end{array}$ & 93 patients & $\begin{array}{l}\text { Choroidal melanoma within } \\
1 \text { - } 2 \text { disc diameters of optic } \\
\text { nerve and at least } 2 \text { disc } \\
\text { diameters from fovea }\end{array}$ & $\begin{array}{l}\text { Mean } 5.5 \\
\text { years }\end{array}$ & -- & $\begin{array}{l}23 \% \text { at } \\
5 \text { years }\end{array}$ & -- & -- \\
\hline $\begin{array}{l}\text { Mosci et al. } \\
2012[24]\end{array}$ & 72 patients & $\begin{array}{l}\text { T3 and T4 choroidal } \\
\text { melanomas }\end{array}$ & $\begin{array}{l}\text { Mean } 53.4 \\
\text { months }\end{array}$ & -- & $\begin{array}{l}7 / 22(32 \%) \\
\text { at } 5 \text { years }\end{array}$ & -- & -- \\
\hline $\begin{array}{l}\text { Tran et al. } \\
2012 \text { [25] }\end{array}$ & 59 patients & $\begin{array}{l}\text { Peripapillary choroidal } \\
\text { melanoma }\end{array}$ & $\begin{array}{l}\text { Median } 63 \\
\text { months }\end{array}$ & -- & $\begin{array}{l}17 \% \text { at } \\
5 \text { years }\end{array}$ & -- & -- \\
\hline $\begin{array}{l}\text { Riechardt et al. } \\
\quad 2014 \text { [34] }\end{array}$ & 147 patients & $\begin{array}{l}\text { Peripapillary choroidal } \\
\text { melanoma }\end{array}$ & $\begin{array}{l}\text { Mean } 78 \\
\text { months }\end{array}$ & -- & $14 \%$ & -- & -- \\
\hline $\begin{array}{l}\text { Schonfeld et al. } \\
\quad 2014[26]\end{array}$ & 18 patients & $\begin{array}{l}\text { Choroidal melanoma in } \\
\text { intermediate zone of fundus }\end{array}$ & $\begin{array}{l}\text { Median } 77.2 \\
\text { months }\end{array}$ & -- & $66.7 \%$ & -- & -- \\
\hline
\end{tabular}

Table 3. Eye preservation outcomes following plaque therapy for choroidal melanoma.

\begin{tabular}{|c|c|c|c|c|c|c|c|}
\hline Study & Sample Size & Tumor Type & $\begin{array}{l}\text { Duration of } \\
\text { Follow-Up }\end{array}$ & $\begin{array}{l}\text { Number (\%) } \\
\text { Requiring } \\
\text { Enucleation } \\
\text { for Tumor } \\
\text { Recurrence } \\
\text { by End of } \\
\text { Follow-Up }\end{array}$ & $\begin{array}{l}\text { Number (\%) } \\
\text { Requiring } \\
\text { Enucleation } \\
\text { for Treatment } \\
\text { Side Effects } \\
\text { by End of } \\
\text { Follow-Up }\end{array}$ & $\begin{array}{l}\text { Total Number } \\
\text { (\%) Requiring } \\
\text { Enucleation }\end{array}$ & $\begin{array}{c}\text { Predictors of } \\
\text { Requiring } \\
\text { Enucleation } \\
\text { after Treatment }\end{array}$ \\
\hline $\begin{array}{l}\text { Bergman et al. } \\
\quad 2005 \text { [29] }\end{array}$ & 579 eyes & $\begin{array}{l}\text { Choroidal and ciliary } \\
\text { body melanomas }\end{array}$ & $\begin{array}{l}3 \text { and } \\
5 \text { years }\end{array}$ & $\begin{array}{c}60 / 579 \\
(10.4 \%) \\
\text { at } 3 \text { years }\end{array}$ & $\begin{array}{c}17 / 579 \\
(2.9 \%) \\
\text { at } 3 \text { years }\end{array}$ & $\begin{array}{c}106 \text { patients } \\
\text { enucleated total } \\
\text { with cumulative } \\
\text { incidence } 16.8 \%\end{array}$ & -- \\
\hline $\begin{array}{l}\text { Sagoo et al. } \\
2007[10]\end{array}$ & 37 patients & $\begin{array}{c}\text { Circumpapillary } \\
\text { choroidal melanoma }\end{array}$ & $\begin{array}{l}\text { Mean } 52 \\
\text { months, } \\
\text { median } 46 \\
\text { months }\end{array}$ & $\begin{array}{c}3 / 37 \\
(8.1 \%)\end{array}$ & $\begin{array}{c}4 / 37 \\
(10.8 \%)\end{array}$ & $\begin{array}{c}7 / 37 \\
(18.9 \%)\end{array}$ & -- \\
\hline $\begin{array}{l}\text { Sagoo et al. } \\
2008[11]\end{array}$ & 141 patients & $\begin{array}{c}\text { Juxtapapillary choroidal } \\
\text { melanoma overhanging } \\
\text { optic disc }\end{array}$ & $\begin{array}{l}\text { Mean } 56 \\
\text { months }\end{array}$ & $\begin{array}{l}7 / 141 \\
(5.0 \%)\end{array}$ & $\begin{array}{c}20 / 141 \\
(14.2 \%)\end{array}$ & $\begin{array}{c}27 / 141 \\
(19.1 \%)\end{array}$ & -- \\
\hline $\begin{array}{l}\text { Finger et al. } \\
2010 \text { [30] }\end{array}$ & 384 patients & $\begin{array}{c}\text { Uveal melanomas treated } \\
\text { with palladium-103 } \\
\text { brachytherapy }\end{array}$ & $\begin{array}{c}\text { Mean } 47.2 \\
\text { months }\end{array}$ & -- & -- & $\begin{array}{l}11 / 384(2.9 \%) \\
\text { within } 3 \text { years }\end{array}$ & -- \\
\hline $\begin{array}{l}\text { Gunduz et al. } \\
2010[12]\end{array}$ & 24 patients & $\begin{array}{l}\text { Choroidal melanoma } \\
\text { treated with ruthenium } \\
\text { brachytherapy alone }\end{array}$ & -- & -- & -- & $\begin{array}{l}2 / 15(13.3 \%) \text { for } \\
\text { tumors } \leq 5 \mathrm{~mm} \text {, } \\
1 / 9(11.1 \%) \text { for } \\
\text { tumors }>5 \mathrm{~mm} \\
\text { to }<8 \mathrm{~mm}\end{array}$ & -- \\
\hline $\begin{array}{l}\text { Verschueren } \\
\text { et al. } 2010 \text { [13] }\end{array}$ & 430 patients & Choroidal melanoma & $\begin{array}{l}\text { Median } 50 \\
\text { months }\end{array}$ & $\begin{array}{l}10 / 430 \\
(2.3 \%)\end{array}$ & $\begin{array}{c}7 / 430 \\
(1.6 \%)\end{array}$ & $17(4.4 \%)$ & -- \\
\hline
\end{tabular}




\begin{tabular}{|c|c|c|c|c|c|c|c|}
\hline \multicolumn{8}{|l|}{ Continued } \\
\hline $\begin{array}{l}\text { Karlovits et al. } \\
2011[14]\end{array}$ & 35 patients & $\begin{array}{l}\text { Choroidal melanoma } \\
\text { treated with iodine } \\
\text { brachytherapy }\end{array}$ & $\begin{array}{l}\text { Median } 45 \\
\text { months }\end{array}$ & -- & -- & $6 \%$ & -- \\
\hline $\begin{array}{l}\text { Finger et al. } \\
2012[16]\end{array}$ & 24 patients & $\begin{array}{c}\text { Choroidal melanomas } \\
\text { within } 1.5 \text { mm of optic } \\
\text { disc treated with slotted } \\
\text { radiotherapy }\end{array}$ & $\begin{array}{l}\text { Mean } 23 \\
\text { months }\end{array}$ & -- & -- & $0(0.0 \%)$ & -- \\
\hline $\begin{array}{l}\text { Berry et al. } \\
2013 \text { [17] }\end{array}$ & 82 patients & $\begin{array}{c}\text { Medium sized } \\
\text { choroidal melanomas }\end{array}$ & $\begin{array}{l}\text { Median } \\
46.8 \\
\text { months }\end{array}$ & $\begin{array}{c}2 / 82 \\
(2.4 \%)\end{array}$ & -- & -- & -- \\
\hline $\begin{array}{l}\text { Caminal et al. } \\
2013 \text { [18] }\end{array}$ & 54 patients & $\begin{array}{l}\text { Choroidal melanoma } \\
\text { treated with iodine } \\
\text { brachytherapy }\end{array}$ & $\begin{array}{l}\text { Mean } 59.47 \\
\text { months }\end{array}$ & $\begin{array}{c}1 / 54 \\
(1.9 \%)\end{array}$ & $\begin{array}{c}6 / 54 \\
(11.1 \%)\end{array}$ & $\begin{array}{c}7 / 54 \\
(13.0 \%)\end{array}$ & -- \\
\hline $\begin{array}{l}\text { Marconi et al. } \\
2013 \text { [31] }\end{array}$ & 94 patients & $\begin{array}{l}\text { Choroidal melanoma } \\
\text { treated with ruthenium } \\
\text { brachytherapy }\end{array}$ & $\begin{array}{l}\text { Median } 39 \\
\text { months }\end{array}$ & $\begin{array}{c}4 / 94 \\
(4.3 \%)\end{array}$ & $\begin{array}{c}4 / 94 \\
(4.3 \%)\end{array}$ & & -- \\
\hline $\begin{array}{l}\text { Sagoo et al. } \\
2014 \text { [32] }\end{array}$ & 650 eyes & $\begin{array}{c}\text { Juxtapapillary } \\
\text { choroidal melanoma }\end{array}$ & $\begin{array}{l}\text { Median } 40 \\
\text { months }\end{array}$ & -- & -- & $\begin{array}{l}16 \% \text { at } \\
5 \text { years }\end{array}$ & $\begin{array}{l}\text { Plaque size, tumor } \\
\text { recurrence, neovascular } \\
\text { glaucoma, absence } \\
\text { of nonproliferative } \\
\text { retinopathy }\end{array}$ \\
\hline $\begin{array}{c}\text { Semenova et al. } \\
2014[20]\end{array}$ & 47 patients & $\begin{array}{l}\mathrm{T} 3 \text { or } \mathrm{T} 4 \text { sized choroidal } \\
\text { melanomas treated with } \\
\text { palladium brachytherapy }\end{array}$ & $\begin{array}{l}\text { Median } 47 \\
\text { months }\end{array}$ & $\begin{array}{c}3 / 47 \\
(6.4 \%)\end{array}$ & $\begin{array}{c}2 / 47 \\
(4.3 \%)\end{array}$ & -- & -- \\
\hline
\end{tabular}

Table 4. Eye preservation outcomes following proton beam therapy for choroidal melanoma.

\begin{tabular}{|c|c|c|c|c|c|c|c|}
\hline Study & Sample Size & Tumor Type & $\begin{array}{l}\text { Duration of } \\
\text { Follow-Up }\end{array}$ & $\begin{array}{l}\text { Number (\%) } \\
\text { Requiring } \\
\text { Enucleation } \\
\text { for Tumor } \\
\text { Recurrence } \\
\text { by End of } \\
\text { Follow-Up }\end{array}$ & $\begin{array}{l}\text { Number (\%) } \\
\text { Requiring } \\
\text { Enucleation } \\
\text { for Treatment } \\
\text { Side Effects } \\
\text { by End of } \\
\text { Follow-Up }\end{array}$ & $\begin{array}{l}\text { Total Number } \\
\text { (\%) Requiring } \\
\text { Enucleation }\end{array}$ & $\begin{array}{c}\text { Predictors } \\
\text { of Requiring } \\
\text { Enucleation } \\
\text { after } \\
\text { Treatment }\end{array}$ \\
\hline $\begin{array}{c}\text { Damato et al. } \\
2005 \text { [23] }\end{array}$ & 349 patients & $\begin{array}{l}\text { Choroidal } \\
\text { melanoma }\end{array}$ & $\begin{array}{l}\text { Median } 3.1 \\
\text { years }\end{array}$ & $\begin{array}{c}8 / 349 \\
(2.3 \%)\end{array}$ & $\begin{array}{l}17 / 349 \\
(4.9 \%)\end{array}$ & $\begin{array}{l}25 / 349(7.2 \%) \\
1.6 \% \text { at } 1 \text { year, } \\
4.0 \% \text { at } 2 \text { years, } \\
9.4 \% \text { at } 5 \text { years }\end{array}$ & -- \\
\hline $\begin{array}{c}\text { Conway et al. } \\
2006 \text { [9] }\end{array}$ & 21 patients & $\begin{array}{c}\text { Extra large choroidal } \\
\text { or ciliochoroidal } \\
\text { melanomas }\end{array}$ & $\begin{array}{l}\text { Median } 28 \\
\text { months }\end{array}$ & $\begin{array}{c}4 / 21 \\
(19.0 \%)\end{array}$ & $\begin{array}{c}6 / 21 \\
(28.6 \%)\end{array}$ & $\begin{array}{c}10 / 21 \\
(47.6 \%)\end{array}$ & -- \\
\hline $\begin{array}{c}\text { Macdonald } \\
\text { et al. } 2011 \\
{[35]}\end{array}$ & 147 patients & Uveal melanoma & $\begin{array}{l}\text { Mean } 4.4 \\
\text { years }\end{array}$ & $\begin{array}{l}48 \% \text { of } \\
\text { enucleations } \\
\text { for suspected } \\
\text { recurrence }\end{array}$ & $\begin{array}{l}52 \% \text { of } \\
\text { enucleations for } \\
\text { complications } \\
\text { from proton } \\
\text { therapy }\end{array}$ & $\begin{array}{c}22.4 \% \text { of } \\
\text { patients received } \\
\text { enucleation }\end{array}$ & -- \\
\hline $\begin{array}{c}\text { Mosci et al. } \\
2012[24]\end{array}$ & 72 patients & $\begin{array}{l}\text { T3 and T4 choroidal } \\
\text { melanomas }\end{array}$ & $\begin{array}{c}\text { Mean } 53.4 \\
\text { months }\end{array}$ & -- & -- & $\begin{array}{l}26 \% \text { of patients } \\
\text { received enculeation } \\
\text { over } 5 \text { years }\end{array}$ & -- \\
\hline $\begin{array}{l}\text { Tran et al. } \\
2012 \text { [25] }\end{array}$ & 59 patients & $\begin{array}{c}\text { Peripapillary } \\
\text { choroidal melanoma }\end{array}$ & $\begin{array}{l}\text { Median } 63 \\
\text { months }\end{array}$ & $\begin{array}{c}4 / 59 \\
(6.8 \%)\end{array}$ & $\begin{array}{c}8 / 59 \\
(13.6 \%)\end{array}$ & $\begin{array}{l}12 / 59 \\
(20 \%)\end{array}$ & -- \\
\hline $\begin{array}{l}\text { Riechardt et al. } \\
\quad 2014 \text { [34] }\end{array}$ & 147 patients & $\begin{array}{c}\text { Peripapillary } \\
\text { choroidal melanoma }\end{array}$ & $\begin{array}{l}\text { Mean } 78 \\
\text { months }\end{array}$ & $\begin{array}{c}6 / 147 \\
(4.1 \%)\end{array}$ & $\begin{array}{c}8 / 147 \\
(5.4 \%)\end{array}$ & $\begin{array}{c}9.5 \% \text { enucleated } \\
\text { at } 5 \text { years, } 10.7 \% \\
\text { enucleated at } \\
10 \text { years }\end{array}$ & -- \\
\hline $\begin{array}{l}\text { Schonfeld et al. } \\
\quad 2014 \text { [26] }\end{array}$ & 18 patients & $\begin{array}{l}\text { Choroidal melanoma } \\
\text { in intermediate } \\
\text { zone of fundus }\end{array}$ & $\begin{array}{l}\text { Median } 77.2 \\
\text { months }\end{array}$ & -- & -- & $\begin{array}{c}1 / 18 \\
(5.6 \%)\end{array}$ & -- \\
\hline
\end{tabular}


rates greater than $20 \%$, which was noticeably higher than rates reported in studies of plaque therapy. One study [9] was limited to extra-large and ciliochoroidal tumors and another [24] to stage T3 and T4 tumors, which might explain the higher rates of enucleation reported in these studies. There were no included studies that examined multivariate predictors of secondary enucleation following proton beam therapy.

\subsection{Optic Neuropathy}

Studies examining optic neuropathy following plaque and proton beam therapy for choroidal melanoma are outlined in Table 5 and Table 6. Optic neuropathy following radiation is thought to be secondary to demyelination and neuronal degeneration following glial and endothelial cell injury from radiation, and most often leads to irreversible vision loss over time [8] [36] [37]. There were ten studies [10]-[13] [16] [17] [19] [20] [32] [38] included in this review that examined optic neuropathy following plaque therapy, with sample sizes ranging from 24 to 650 patients and follow-up time ranging from a mean of 23 months to 56 months. Rates of radiation optic neuropathy ranged from $1.6 \%$ to $62.5 \%$, with the highest rates being in studies of choroidal melanoma within $1.5 \mathrm{~mm}$ of the optic disc [24] and juxtapapillary choroidal melanoma [32]. In the two studies that examined multivariate predictors of optic neuropathy [19] [32], plaque size and subfoveal or juxtapapillary tumor location were found to be predictive of optic neuropathy following plaque therapy.

There were three studies reviewed [9] [33] [34] that examined optic neuropathy following proton beam therapy for choroidal melanoma. Sample sizes ranged from 21 to 384 patients and follow-up time ranged from a median of 28 months to a mean of 78 months. Similar to the studies of neuropathy following plaque therapy, the two studies of optic neuropathy following proton beam therapy, which focused on peripapillary tumors [33] [34], found high rates of neuropathy following treatment (89.6\% and 67.7\%), and proximity of the tumor to the optic disc was found to be predictive of radiation optic neuropathy in one of these studies [33].

Table 5. Optic neuropathy outcomes following plaque therapy for choroidal melanoma.

\begin{tabular}{|c|c|c|c|c|c|}
\hline Study & Sample Size & Tumor Type & $\begin{array}{l}\text { Duration of } \\
\text { Follow-Up }\end{array}$ & $\begin{array}{l}\text { Number (\%) with } \\
\text { Optic Neuropathy at } \\
\text { End of Follow-Up }\end{array}$ & $\begin{array}{c}\text { Predictors of Optic } \\
\text { Neuropathy after } \\
\text { Treatment }\end{array}$ \\
\hline $\begin{array}{l}\text { Sagoo et al. } \\
2007[10]\end{array}$ & 37 patients & $\begin{array}{l}\text { Circumpapillary choroidal } \\
\text { melanoma }\end{array}$ & $\begin{array}{l}\text { Mean } 52 \text { months, } \\
\text { median } 46 \text { months }\end{array}$ & 9/37 (24.3\%) & -- \\
\hline $\begin{array}{l}\text { Sagoo et al. } \\
2008[11]\end{array}$ & 141 patients & $\begin{array}{l}\text { Juxtapapillary choroidal } \\
\text { melanoma overhanging optic disc }\end{array}$ & Mean 56 months & $57 / 141(40.4 \%)$ & -- \\
\hline $\begin{array}{l}\text { Gunduz et al. } \\
2010[12]\end{array}$ & 24 patients & $\begin{array}{l}\text { Choroidal melanoma treated with } \\
\text { ruthenium brachytherapy alone }\end{array}$ & -- & $1 / 24(4.1 \%)$ & -- \\
\hline $\begin{array}{l}\text { Verschueren } \\
\text { et al. } 2010 \text { [13] }\end{array}$ & 430 patients & Choroidal melanoma & Median 50 months & $7 / 430(1.6 \%)$ & -- \\
\hline $\begin{array}{l}\text { Finger et al. } \\
2012[16]\end{array}$ & 24 patients & $\begin{array}{l}\text { Choroidal melanomas within } \\
1.5 \mathrm{~mm} \text { of optic disc treated } \\
\text { with slotted radiotherapy }\end{array}$ & Mean 23 months & $15 / 24(62.5 \%)$ & -- \\
\hline $\begin{array}{c}\text { Berry et al. } \\
2013 \text { [17] }\end{array}$ & 82 patients & $\begin{array}{l}\text { Medium sized choroidal } \\
\text { melanomas }\end{array}$ & Median 46.8 months & 12/82 (14.6\%) & -- \\
\hline $\begin{array}{l}\text { Krema et al. } \\
2013 \text { [38] }\end{array}$ & 30 patients & $\begin{array}{c}\text { Posterior tumor margin } \\
0-2.5 \mathrm{~mm} \text { from optic disc }\end{array}$ & -- & $40 \%$ & -- \\
\hline $\begin{array}{l}\text { Semenova et al. } \\
2013 \text { [19] }\end{array}$ & 72 patients & $\begin{array}{l}\text { Small choroidal } \\
\text { melanomas }\end{array}$ & Mean 54 months & 15/72 (20.8\%) & $\begin{array}{l}\text { Subfoveal or } \\
\text { juxtapapillary } \\
\text { tumor location }\end{array}$ \\
\hline $\begin{array}{l}\text { Sagoo et al. } \\
2014[32]\end{array}$ & 650 eyes & $\begin{array}{l}\text { Juxtapapillary choroidal } \\
\text { melanoma }\end{array}$ & Median 40 months & $61 \%$ at 5 years & Plaque size \\
\hline $\begin{array}{l}\text { Semenova et al. } \\
2014[20]\end{array}$ & 47 patients & $\begin{array}{l}\text { T3 or T4 sized choroidal } \\
\text { melanomas treated with } \\
\text { palladium brachytherapy }\end{array}$ & Median 47 months & $24 / 47$ (51\%) & -- \\
\hline
\end{tabular}


Table 6. Optic neuropathy outcomes following proton beam therapy for choroidal melanoma.

\begin{tabular}{|c|c|c|c|c|c|}
\hline Study & Sample Size & Tumor Type & $\begin{array}{l}\text { Duration of } \\
\text { Follow-Up }\end{array}$ & $\begin{array}{l}\text { Number (\%) with } \\
\text { Optic Neuropathy at } \\
\text { End of Follow-Up }\end{array}$ & $\begin{array}{c}\text { Predictors of Optic } \\
\text { Neuropathy after } \\
\text { Treatment }\end{array}$ \\
\hline $\begin{array}{c}\text { Conway et al. } \\
2006 \text { [9] }\end{array}$ & 21 patients & $\begin{array}{l}\text { Extra large choroidal or } \\
\text { ciliochoroidal melanomas }\end{array}$ & Median 28 months & 2/21 (9.5\%) & -- \\
\hline $\begin{array}{l}\text { Kim et al. } \\
2010 \text { [33] }\end{array}$ & 93 patients & $\begin{array}{l}\text { Choroidal melanoma within } \\
1 \text { - } 2 \text { disc diameters of optic } \\
\text { nerve and at least } 2 \text { disc } \\
\text { diameters from fovea }\end{array}$ & Mean 5.5 years & 63/93 (67.7\%) & $\begin{array}{l}\text { Proximity to } \\
\text { optic disc }\end{array}$ \\
\hline $\begin{array}{l}\text { Riechardt et al. } \\
\quad 2014 \text { [34] }\end{array}$ & 147 patients & $\begin{array}{c}\text { Peripapillary } \\
\text { choroidal melanoma }\end{array}$ & Mean 78 months & $89.6 \%$ at 5 years & -- \\
\hline
\end{tabular}

\subsection{Radiation Retinopathy}

Studies examining radiation retinopathy following plaque and proton beam therapy for choroidal melanoma are outlined in Table 7 and Table 8. Radiation retinopathy is characterized by retinal hemorrhage, exudation, edema, ischemia and neovascularization of the retina. These abnormalities may lead to permanent visual loss [39] [40]. There were 13 included studies [10] [11] [13] [15]-[17] [19] [20] [30] [32] [38] [41] [42] that examined radiation retinopathy following plaque therapy, with sample sizes ranging from 24 to 3841 patients and follow-up time ranging from 23 to 56 months. Rates of maculopathy ranged from $8 \%$ to 56\%, with the highest rates reported in studies of tumors near the optic disc [16] [32]. Similarly, there was a wide range of retinopathy reported $(3.6 \%$ - 66\%), but higher rates were reported in studies of tumors near the optic disc (60\% - 66\%) [32] [38] and in large or subfoveal tumors (56\% - 66\%) [15] [20]. Tumor location was the only predictive factor reported in more than one study [30] [42].

There were two included studies [9] [34] that examined radiation retinopathy following proton beam therapy. While these studies differed greatly in sample size and follow-up time, the study that focused on juxtapapillary tumors [34] also found a high rate of retinopathy (90.3\%) following proton beam therapy, similar to the studies of juxtapapillary tumors treated with plaque therapy. The other study [9], which focused on large tumors, found a low rate of retinopathy compared to the studies of retinopathy following plaque therapy, suggesting that tumor size may not be related to the development of retinopathy for patients receiving proton beam therapy.

\subsection{Neovascular Glaucoma}

Studies examining neovascular glaucoma after plaque and proton beam therapy for choroidal melanoma are outlined in Table 9 and Table 10. Neovascularization of the retina and the iris is frequently a result of retinal ischemia from radiation retinopathy, leading to elevated intraocular pressure and permanent visual field loss from glaucoma [43]. There were seven studies [10] [11] [16] [20] [31] [32] [38] that examined neovascular glaucoma following plaque therapy for choroidal melanoma. Sample sizes ranged from 24 to 650 patients and follow-up time ranged from a mean of 23 months to 56 months. Rates of neovascular glaucoma in small and medium tumors were low $(0.0 \%-3 \%)$ [16] [31], while studies of tumors near the optic nerve and large tumors reported slightly higher rates (8\% - 17\%) [10] [11] [20] [32] [38]. One study found that tumor thickness was predictive of neovascular glaucoma [32].

There were three studies of neovascular glaucoma following proton beam therapy for choroidal melanoma [9] [25] [26], with sample sizes ranging from 18 to 59 patients and follow-up time ranging from a median of 28 to 77.2 months. While reported rates of neovascular glaucoma were higher in all of these studies $(23 \%-38.1 \%)$ compared to those of neovascular glaucoma following plaque therapy, two of these studies focused on large tumors and those near the optic nerve. The one study [9] that examined predictors of neovascular glaucoma after proton beam therapy also found that tumor thickness and proximity to the optic nerve were predictive of neovascular glaucoma.

\subsection{Cataract}

Studies examining cataract formation following plaque and proton beam therapy for choroidal melanoma are 
Table 7. Radiation retinopathy outcomes following plaque therapy for choroidal melanoma.

\begin{tabular}{|c|c|c|c|c|c|}
\hline Study & Sample Size & Tumor Type & $\begin{array}{l}\text { Duration of } \\
\text { Follow-Up }\end{array}$ & $\begin{array}{c}\text { Number (\%) with Radiation } \\
\text { Retinopathy at End of } \\
\text { Follow-Up }\end{array}$ & $\begin{array}{c}\text { Predictors of Radiation } \\
\text { Retinopathy after } \\
\text { Treatment }\end{array}$ \\
\hline $\begin{array}{l}\text { Sagoo et al. } \\
2007[10]\end{array}$ & 37 patients & $\begin{array}{c}\text { Circumpapillary } \\
\text { choroidal melanoma }\end{array}$ & $\begin{array}{l}\text { Mean } 52 \\
\text { months, median } \\
46 \text { months }\end{array}$ & $\begin{array}{l}\text { 7/37 (18.9\%) with } \\
\text { proliferative retinopathy }\end{array}$ & -- \\
\hline $\begin{array}{l}\text { Sagoo et al. } \\
2008[11]\end{array}$ & 141 patients & $\begin{array}{c}\text { Juxtapapillary choroidal } \\
\text { melanoma overhanging } \\
\text { optic disc }\end{array}$ & $\begin{array}{l}\text { Mean } 56 \\
\text { months }\end{array}$ & $\begin{array}{l}61(43.3 \%) \text { patients } \\
\text { with nonproliferative } \\
\text { retinopathy, } 26 \text { patients } \\
(18.4 \%) \text { progressed to } \\
\text { proliferative retinopathy } \\
\text { by the end of follow-up }\end{array}$ & -- \\
\hline $\begin{array}{l}\text { Correa et al. } \\
2009[41]\end{array}$ & 120 patients & $\begin{array}{c}\text { Choroidal melanoma } \\
\text { treated with iodine-125 } \\
\text { brachytherapy }\end{array}$ & -- & $7.5 \%$ with retinopathy & -- \\
\hline $\begin{array}{l}\text { Bianciotto et al. } \\
\quad 2010 \text { [42] }\end{array}$ & 3841 patients & Choroidal melanoma & -- & $\begin{array}{l}\text { 138/3841 (3.6\%) with } \\
\text { proliferative retinopathy }\end{array}$ & $\begin{array}{l}\text { Young age, diabetes } \\
\text { mellitus, shorter tumor } \\
\text { distance to optic disc }\end{array}$ \\
\hline $\begin{array}{l}\text { Finger et al. } \\
2010[30]\end{array}$ & 384 patients & $\begin{array}{l}\text { Uveal melanomas } \\
\text { treated with } \\
\text { Palladium-103 }\end{array}$ & $\begin{array}{l}\text { Mean } 47.2 \\
\text { months }\end{array}$ & $\begin{array}{l}\text { Maculopathy in } 8 \% \text { of } \\
\text { patients with anterior } \\
\text { tumor and } 41 \% \text { of patients } \\
\text { with posterior tumor }\end{array}$ & $\begin{array}{l}\text { Posterior location, } \\
\text { tumor height }>6.0 \mathrm{~mm}\end{array}$ \\
\hline $\begin{array}{l}\text { Verschueren } \\
\text { et al. } 2010 \text { [13] }\end{array}$ & 430 patients & Choroidal melanoma & $\begin{array}{l}\text { Median } 50 \\
\text { months }\end{array}$ & $\begin{array}{l}\text { 56/430 (13.0\%) with } \\
\text { retinopathy; 65/430 } \\
\text { (15.1\%) with } \\
\text { maculopathy }\end{array}$ & -- \\
\hline $\begin{array}{l}\text { Newman et al. } \\
2011[15]\end{array}$ & 50 patients & $\begin{array}{l}\text { Subfoveal choroidal } \\
\text { melanomas with } \geq 6 \\
\text { months of follow-up }\end{array}$ & $\begin{array}{l}\text { Median } 54 \\
\text { months }\end{array}$ & $\begin{array}{l}28 / 50(56 \%) \text { with } \\
\text { retinopathy; Stage 1: None; } \\
\text { Stage 2: } 14 \% \text {; Stage 3: 54\%; } \\
\text { Stage 4: } 32 \%\end{array}$ & -- \\
\hline $\begin{array}{l}\text { Finger et al. } \\
2012[16]\end{array}$ & 24 patients & $\begin{array}{c}\text { Choroidal melanomas } \\
\text { within } 1.5 \text { mm of optic } \\
\text { disc treated with slotted } \\
\text { radiotherapy }\end{array}$ & $\begin{array}{l}\text { Mean } 23 \\
\text { months }\end{array}$ & $\begin{array}{l}\text { 9/24 (37.5\%) with } \\
\text { maculopathy }\end{array}$ & -- \\
\hline $\begin{array}{c}\text { Berry et al. } \\
2013 \text { [17] }\end{array}$ & 82 patients & $\begin{array}{l}\text { Medium sized choroidal } \\
\text { melanomas }\end{array}$ & $\begin{array}{l}\text { Median } 46.8 \\
\text { months }\end{array}$ & $\begin{array}{l}\text { 31/82 }(37.8 \%) \text { with } \\
\text { retinopathy }\end{array}$ & -- \\
\hline $\begin{array}{l}\text { Krema et al. } \\
2013 \text { [38] }\end{array}$ & 30 patients & $\begin{array}{l}\text { Posterior tumor } \\
\text { margin } 0-2.5 \mathrm{~mm} \\
\text { from optic disc }\end{array}$ & -- & $60 \%$ with retinopathy & -- \\
\hline $\begin{array}{c}\text { Semenova } \\
\text { et al. } 2013 \text { [19] }\end{array}$ & 72 patients & $\begin{array}{l}\text { Small choroidal } \\
\text { melanomas }\end{array}$ & $\begin{array}{l}\text { Mean } 54 \\
\text { months }\end{array}$ & $\begin{array}{l}\text { 31/72 (43.1\%) with } \\
\text { maculopathy }\end{array}$ & -- \\
\hline $\begin{array}{l}\text { Sagoo et al. } \\
2014[32]\end{array}$ & 650 eyes & $\begin{array}{c}\text { Juxtapapillary } \\
\text { choroidal melanoma }\end{array}$ & $\begin{array}{l}\text { Median } 40 \\
\text { months }\end{array}$ & $\begin{array}{l}\text { Nonproliferative } \\
\text { retinopathy at } 5 \text { years: } \\
66 \% \text {; Proliferative } \\
\text { retinopathy at } 5 \text { years: } \\
\text { 24\%; Maculopathy at } \\
5 \text { years: } 56 \%\end{array}$ & $\begin{array}{c}\text { Nonproliferative: } \\
\text { Hypertension; Proliferative: } \\
\text { Mean tumor thickness; } \\
\text { Maculopathy: Visual } \\
\text { acuity > 20/60 at } \\
\text { presentation }\end{array}$ \\
\hline $\begin{array}{c}\text { Semenova } \\
\text { et al. } 2014 \text { [20] }\end{array}$ & 47 patients & $\begin{array}{l}\text { T3 or T4 sized choroidal } \\
\text { melanomas treated with } \\
\text { palladium brachytherapy }\end{array}$ & $\begin{array}{l}\text { Median } 47 \\
\text { months }\end{array}$ & $\begin{array}{l}\text { 31/47 }(66 \%) \text { with } \\
\text { retinopathy }\end{array}$ & -- \\
\hline
\end{tabular}


Table 8. Radiation retinopathy outcomes following proton beam therapy for choroidal melanoma.

\begin{tabular}{cccccc}
\hline Study & Sample Size & Tumor Type & $\begin{array}{c}\text { Duration of } \\
\text { Follow-Up }\end{array}$ & $\begin{array}{c}\text { Number (\%) with Radiation } \\
\text { Retinopathy at End of } \\
\text { Follow-Up }\end{array}$ & $\begin{array}{c}\text { Predictors of Radiation } \\
\text { Retinopathy after } \\
\text { Treatment }\end{array}$ \\
\hline $\begin{array}{c}\text { Conway } \text { et al. } \\
2006 \text { [9] }\end{array}$ & 21 patients & $\begin{array}{c}\text { Extra large choroidal or } \\
\text { ciliochoroidal melanomas }\end{array}$ & $\begin{array}{c}\text { Median } 28 \\
\text { months }\end{array}$ & $2 / 21(9.5 \%)$ & -- \\
$\begin{array}{c}\text { Riechardt } \text { et al. } \\
2014[34]\end{array}$ & 147 patients & $\begin{array}{c}\text { Peripapillary choroidal } \\
\text { melanoma }\end{array}$ & $\begin{array}{c}\text { Mean } 78 \\
\text { months }\end{array}$ & $90.3 \%$ at 5 years & - \\
\hline
\end{tabular}

Table 9. Neovascular glaucoma outcomes following plaque therapy for choroidal melanoma.

\begin{tabular}{|c|c|c|c|c|c|}
\hline Study & Sample Size & Tumor Type & $\begin{array}{l}\text { Duration of } \\
\text { Follow-Up }\end{array}$ & $\begin{array}{l}\text { Number (\%) with } \\
\text { Neovascular Glaucoma } \\
\text { at End of Follow-Up }\end{array}$ & $\begin{array}{c}\text { Predictors of } \\
\text { Neovascular Glaucoma } \\
\text { after Treatment }\end{array}$ \\
\hline $\begin{array}{l}\text { Sagoo et al. } \\
2007[10]\end{array}$ & 37 patients & $\begin{array}{l}\text { Circumpapillary choroidal } \\
\text { melanoma }\end{array}$ & $\begin{array}{l}\text { Mean } 52 \text { months, } \\
\text { median } 46 \text { months }\end{array}$ & 5/37 (13.5\%) & -- \\
\hline $\begin{array}{l}\text { Sagoo et al. } \\
2008[11]\end{array}$ & 141 patients & $\begin{array}{c}\text { Juxtapapillary choroidal } \\
\text { melanoma overhanging } \\
\text { optic disc }\end{array}$ & $\begin{array}{l}\text { Mean } 56 \\
\text { months }\end{array}$ & 24/141 (17.0\%) & -- \\
\hline $\begin{array}{l}\text { Finger et al. } \\
2012[16]\end{array}$ & 24 patients & $\begin{array}{c}\text { Choroidal melanomas } \\
\text { within } 1.5 \mathrm{~mm} \text { of optic } \\
\text { disc treated with slotted } \\
\text { radiotherapy }\end{array}$ & $\begin{array}{l}\text { Mean } 23 \\
\text { months }\end{array}$ & $0 / 24(0.0 \%)$ & -- \\
\hline $\begin{array}{l}\text { Krema et al. } \\
2013 \text { [38] }\end{array}$ & 30 patients & $\begin{array}{c}\text { Posterior tumor margin } \\
0 \text { - } 2.5 \mathrm{~mm} \text { from optic disc }\end{array}$ & & $8 \%$ & -- \\
\hline $\begin{array}{l}\text { Marconi et al. } \\
2013[31]\end{array}$ & 94 patients & $\begin{array}{l}\text { Choroidal melanoma } \\
\text { treated with ruthenium } \\
\text { brachytherapy }\end{array}$ & $\begin{array}{l}\text { Median } 39 \\
\text { months }\end{array}$ & $\begin{array}{l}\text { 3\% with secondary } \\
\text { glaucoma }\end{array}$ & -- \\
\hline $\begin{array}{l}\text { Sagoo et al. } \\
2014 \text { [32] }\end{array}$ & 650 eyes & $\begin{array}{l}\text { Juxtapapillary choroidal } \\
\text { melanoma }\end{array}$ & $\begin{array}{l}\text { Median } 40 \\
\text { months }\end{array}$ & $15 \%$ at 5 years & Mean tumor thickness \\
\hline $\begin{array}{l}\text { Semenova et al. } \\
\quad 2014 \text { [20] }\end{array}$ & 47 patients & $\begin{array}{l}\mathrm{T} 3 \text { or } \mathrm{T} 4 \text { sized choroidal } \\
\text { melanomas treated with } \\
\text { palladium brachytherapy }\end{array}$ & $\begin{array}{l}\text { Median } 47 \\
\text { months }\end{array}$ & $\begin{array}{c}\text { 8/47 (17\%) with } \\
\text { secondary glaucoma }\end{array}$ & -- \\
\hline
\end{tabular}

Table 10. Neovascular glaucoma outcomes following proton beam therapy for choroidal melanoma.

\begin{tabular}{|c|c|c|c|c|c|}
\hline Study & Sample Size & Tumor Type & $\begin{array}{l}\text { Duration of } \\
\text { Follow-Up }\end{array}$ & $\begin{array}{l}\text { Number (\%) with } \\
\text { Neovascular Glaucoma } \\
\text { at End of Follow-Up }\end{array}$ & $\begin{array}{c}\text { Predictors of } \\
\text { Neovascular Glaucoma } \\
\text { after Treatment }\end{array}$ \\
\hline $\begin{array}{c}\text { Conway et al. } \\
2006 \text { [9] }\end{array}$ & 21 patients & $\begin{array}{l}\text { Extra large choroidal or } \\
\text { ciliochoroidal melanomas }\end{array}$ & $\begin{array}{l}\text { Median } 28 \\
\text { months }\end{array}$ & 8/21 (38.1\%) & $\begin{array}{l}\text { Proximity to optic nerve, } \\
\text { tumor thickness }\end{array}$ \\
\hline $\begin{array}{l}\text { Tran et al. } \\
2012[25]\end{array}$ & 59 patients & $\begin{array}{l}\text { Peripapillary choroidal } \\
\text { melanoma }\end{array}$ & $\begin{array}{l}\text { Median } 63 \\
\text { months }\end{array}$ & $31 \%$ at 5 years & -- \\
\hline $\begin{array}{c}\text { Schonfeld } \\
\text { et al. } 2014 \text { [26] }\end{array}$ & 18 patients & $\begin{array}{l}\text { Choroidal melanoma } \\
\text { in intermediate zone } \\
\text { of fundus }\end{array}$ & $\begin{array}{l}\text { Median } 77.2 \\
\text { months }\end{array}$ & $\begin{array}{c}\text { 4/17 (23.5\%) with } \\
\text { secondary glaucoma }\end{array}$ & -- \\
\hline
\end{tabular}

outlined in Table 11 and Table 12. Cataract formation is a well-known complication resulting from radiationinduced opacification of the lens [44]. There were 12 studies [10]-[12] [15]-[17] [20] [31] [38] [41] [44] [45] included that examined cataract development following plaque therapy for choroidal melanoma. Sample sizes ranged from 24 to 532 patients and follow-up time ranged from a mean of 23 months to five years. There was a range of reported cataract development rates from $5.5 \%$ to $68 \%$. The highest rate was reported in patients with 
Table 11. Cataract outcomes following plaque therapy for choroidal melanoma.

\begin{tabular}{|c|c|c|c|c|c|}
\hline Study & Sample Size & Tumor Type & $\begin{array}{l}\text { Duration of } \\
\text { Follow-Up }\end{array}$ & $\begin{array}{l}\text { Number (\%) with } \\
\text { Cataract at End of } \\
\text { Follow-Up }\end{array}$ & $\begin{array}{l}\text { Predictors of } \\
\text { Cataract after } \\
\text { Treatment }\end{array}$ \\
\hline $\begin{array}{l}\text { Collaborative Ocular } \\
\text { Melanoma Study } \\
\text { Group } 2007 \text { [46] }\end{array}$ & 532 patients & $\begin{array}{l}\text { Choroidal melanoma randomized } \\
\text { to iodine-125 brachytherapy }\end{array}$ & 5 years & $\begin{array}{c}362 / 568(68 \%) \text { with } \\
\text { cataract at } 5 \text { years, } \\
49(9 \%) \text { with } \\
\text { cataract surgery }\end{array}$ & Radiation dose \\
\hline $\begin{array}{l}\text { Sagoo et al. } \\
2007[10]\end{array}$ & 37 patients & $\begin{array}{l}\text { Circumpapillary } \\
\text { choroidal melanoma }\end{array}$ & $\begin{array}{l}\text { Mean } 52 \text { months, } \\
\text { median } 46 \text { months }\end{array}$ & 10/37 (27.0\%) & -- \\
\hline $\begin{array}{l}\text { Sagoo et al. } \\
2008 \text { [11] }\end{array}$ & 141 patients & $\begin{array}{l}\text { Juxtapapillary choroidal melanoma } \\
\text { overhanging optic disc }\end{array}$ & Mean 56 months & $66 / 141(46.8 \%)$ & -- \\
\hline $\begin{array}{l}\text { Correa et al. } \\
2009[43]\end{array}$ & 120 patients & $\begin{array}{l}\text { Choroidal melanoma treated } \\
\text { with iodine-125 brachytherapy }\end{array}$ & -- & $31.6 \%$ & -- \\
\hline $\begin{array}{l}\text { Gunduz et al. } \\
2010 \text { [12] }\end{array}$ & 24 patients & $\begin{array}{l}\text { Choroidal melanoma treated with } \\
\text { ruthenium brachytherapy alone }\end{array}$ & -- & 2/24 (8.3\%) & -- \\
\hline $\begin{array}{l}\text { Finger et al. } \\
2011[47]\end{array}$ & $\begin{array}{l}208 \text { phakic } \\
\text { patients }\end{array}$ & $\begin{array}{l}\text { Uveal melanoma treated with } \\
\text { palladium } 103\end{array}$ & $\begin{array}{l}\text { Mean } 39.8 \\
\text { months }\end{array}$ & 76/282 (27.0\%) & $\begin{array}{l}\text { Age, higher } \\
\text { radiation dose }\end{array}$ \\
\hline $\begin{array}{l}\text { Newman et al. } \\
\quad 2011[15]\end{array}$ & 50 patients & $\begin{array}{l}\text { Subfoveal choroidal melanomas } \\
\text { with } \geq 6 \text { months of follow-up }\end{array}$ & $\begin{array}{l}\text { Median } 54 \\
\text { months }\end{array}$ & $\begin{array}{c}22 \% \text { of } 46 \\
\text { patients }\end{array}$ & -- \\
\hline $\begin{array}{l}\text { Finger et al. } \\
2012[16]\end{array}$ & 24 patients & $\begin{array}{l}\text { Choroidal melanomas within } \\
1.5 \mathrm{~mm} \text { of optic disc treated } \\
\text { with slotted radiotherapy }\end{array}$ & $\begin{array}{l}\text { Mean } 23 \\
\text { months }\end{array}$ & 1/18 (5.5\%) & -- \\
\hline $\begin{array}{l}\text { Berry et al. } \\
2013 \text { [17] }\end{array}$ & 82 patients & $\begin{array}{l}\text { Medium sized choroidal } \\
\text { melanomas }\end{array}$ & $\begin{array}{l}\text { Median } 46.8 \\
\text { months }\end{array}$ & 26/82 (31.7\%) & -- \\
\hline $\begin{array}{l}\text { Marconi et al. } \\
\quad 2013 \text { [31] }\end{array}$ & 94 patients & $\begin{array}{l}\text { Choroidal melanoma treated with } \\
\text { ruthenium brachytherapy }\end{array}$ & $\begin{array}{l}\text { Median } 39 \\
\text { months }\end{array}$ & $16 \%$ & -- \\
\hline $\begin{array}{l}\text { Krema et al. } \\
2013 \text { [38] }\end{array}$ & 30 patients & $\begin{array}{c}\text { Posterior tumor margin } \\
0 \text { - } 2.5 \mathrm{~mm} \text { from optic disc }\end{array}$ & & $62 \%$ & -- \\
\hline $\begin{array}{l}\text { Semenova et al. } \\
\quad 2014[20]\end{array}$ & 47 patients & $\begin{array}{l}\mathrm{T} 3 \text { or } \mathrm{T} 4 \text { sized choroidal } \\
\text { melanomas treated with } \\
\text { palladium brachytherapy }\end{array}$ & $\begin{array}{l}\text { Median } 47 \\
\text { months }\end{array}$ & $16 / 47(36 \%)$ & -- \\
\hline
\end{tabular}

Table 12. Cataract outcomes following proton beam therapy for choroidal melanoma.

\begin{tabular}{|c|c|c|c|c|c|}
\hline Study & Sample Size & Tumor Type & $\begin{array}{l}\text { Duration of } \\
\text { Follow-Up }\end{array}$ & $\begin{array}{l}\text { Number (\%) with } \\
\text { Cataract at End } \\
\text { of Follow-Up }\end{array}$ & $\begin{array}{c}\text { Predictors of } \\
\text { Cataract after } \\
\text { Treatment }\end{array}$ \\
\hline $\begin{array}{c}\text { Conway et al. } \\
2006 \text { [9] }\end{array}$ & 21 patients & $\begin{array}{l}\text { Extra large choroidal or } \\
\text { ciliochoroidal melanomas }\end{array}$ & $\begin{array}{l}\text { Median } 28 \\
\text { months }\end{array}$ & 6/21 (28.6\%) & $\begin{array}{l}\text { Tumor far } \\
\text { from fovea }\end{array}$ \\
\hline $\begin{array}{l}\text { Riechardt et al. } \\
\quad 2014 \text { [34] }\end{array}$ & 147 patients & $\begin{array}{l}\text { Peripapillary choroidal } \\
\text { melanoma }\end{array}$ & $\begin{array}{l}\text { Mean } 78 \\
\text { months }\end{array}$ & $\begin{array}{c}31 \% \text { with new cataract } \\
\text { after } 5 \text { years }\end{array}$ & -- \\
\hline
\end{tabular}

choroidal melanoma randomized to iodine-125 brachytherapy in the COMS [44]; the high rate may be explained by the larger sample size, or more accurate documentation of outcomes due to the study being a randomized trial. Another study that reported similar rates by Krema et al. [38] focused on posterior tumors near the optic disc. Of the two studies that examined predictors of cataract formation following plaque therapy [44] [45], both found that radiation dose was predictive of cataract formation following treatment.

There were two studies [9] [34] that examined cataract formation following proton beam therapy for choroidal 
melanoma. While the two studies had very different sample sizes and duration of follow-up and focused on different tumor types, both found similar rates of cataract development following proton beam therapy (28.6\% and $31 \%)$. One of the studies [9] found that tumor distance from the fovea was predictive of cataract development following proton beam therapy, and the other study did not examine predictors of cataract development.

\subsection{Distant Metastasis}

Studies examining distant metastasis from choroidal melanoma following plaque and proton therapy are outlined in Table 13 and Table 14. Distant metastasis from choroidal melanoma is associated with a poor prognosis with a median survival time of less than one year following metastasis [48]. The most common site of metastasis is the liver, with other sites being lung, bone, and skin [49]. There were 13 included studies [10]-[22] that examined the development of distant metastasis following treatment of choroidal melanoma with plaque therapy. Sample sizes ranged from 24 to 650 patients, and follow-up time ranged from a median of 17.3 months to a mean of 59.47 months. Rates of metastasis ranged from $0.0 \%$ to $31.9 \%$, with the highest rates reported in a study of large tumors [20] and in the study with the longest duration of follow-up [18]. Of the studies that reported the time to development of metastasis [13] [14] [17], the average time ranged from 30.4 months [13] to 58 months [14]. Three studies [13] [17] [18] reported rates of metastasis-free survival, with all reported rates greater than $75 \%$. The only study [22] to examine predictors of metastasis found that higher intraocular pressure and greater tumor base were predictive of distant metastasis following plaque therapy.

There were five studies [9] [23]-[26] that examined the development of metastasis following proton therapy for choroidal melanoma. Sample sizes ranged from 21 - 349 patients, and duration of follow-up ranged from a median of 28 months to 77.2 months. Rates of metastasis were similar to those reported after plaque therapy, ranging from $5.6 \%$ to $25.4 \%$. The only study [25] that examined time to metastasis reported a median of 38 months to metastasis, and the studies [24] [25] that examined metastasis-free survival reported survival rates of $72 \%$ to $82 \%$ at five years. Tumor dimension was the only factor reported to be related to metastasis development in more than one study [9] [23].

\section{Summary and Limitations}

This review described the rates of a variety of complications following treatment with plaque versus proton beam therapy for patients with choroidal melanoma. In our descriptive comparisons of the rates of complications following the two different treatment modalities, patients with proton beam therapy have potentially higher rates of vision loss, enucleation, and neovascular glaucoma after treatment when compared to patients with plaque therapy. Reported rates of optic neuropathy, radiation retinopathy, and cataract formation were widely variable for the two treatment modalities, with no consistent differences that could be ascertained when trying to compare the two types of therapy. Reported rates of metastasis and metastasis-free survival appeared similar in studies of plaque versus proton beam therapy, though comparisons were limited by study population, tumor type, and duration of follow-up. The most common reported predictors of ocular complications following both types of therapy were tumor distance from the optic nerve, tumor thickness, and radiation dose, suggesting that inherent tumor characteristics play a role in visual prognosis.

This review was limited mainly by differences in study design, patient inclusion criteria, and the limited number of studies available. There were wide ranges of sample sizes and follow-up times which made it inherently difficult to compare complication rates across studies for both types of treatment. Additionally, several studies focused on specific tumor types such as large tumors or those near the optic nerve, which may have affected the complication rates reported in those studies. Finally, several studies included in this review only examined visual complications as a secondary outcome and did not provide comprehensive information regarding these complications. For further understanding of the side effects of plaque and proton beam therapy for choroidal melanoma, an aggregate registry of ocular and systemic complications of choroidal melanoma treatment would be beneficial.

In conclusion, both plaque and proton beam therapy are associated with a wide range of potentially devastating complications for patients with choroidal melanoma, and proton beam therapy may be associated with higher rates of vision loss, enucleation, and neovascular glaucoma. In addition to the development of treatments that prolong remission and survival, further studies are needed to compare outcomes and quality of life in patients receiving plaque versus proton beam therapy for choroidal melanoma. 
Table 13. Distant metastasis outcomes following plaque therapy for choroidal melanoma.

\begin{tabular}{|c|c|c|c|c|c|c|c|}
\hline Study & Sample Size & Tumor Type & $\begin{array}{l}\text { Duration of } \\
\text { Follow-Up }\end{array}$ & $\begin{array}{c}\text { Number (\%) } \\
\text { with Distant } \\
\text { Metastasis } \\
\text { at End of } \\
\text { Follow-Up }\end{array}$ & $\begin{array}{l}\text { Mean Time } \\
\text { to Metastasis }\end{array}$ & $\begin{array}{l}\text { Percentage with } \\
\text { Metastasis-Free } \\
\quad \text { Survival }\end{array}$ & $\begin{array}{l}\text { Predictors } \\
\text { of Distant } \\
\text { Metastasis } \\
\text { after } \\
\text { Treatment }\end{array}$ \\
\hline $\begin{array}{l}\text { Sagoo et al. } \\
2007 \text { [10] }\end{array}$ & 37 patients & $\begin{array}{l}\text { Circumpapillary } \\
\text { choroidal melanoma }\end{array}$ & $\begin{array}{l}\text { Mean } 52 \\
\text { months, median } \\
46 \text { months }\end{array}$ & $1 / 37$ (4\%) & -- & -- & -- \\
\hline $\begin{array}{l}\text { Sagoo et al. } \\
2008 \text { [11] }\end{array}$ & 141 patients & $\begin{array}{l}\text { Juxtapapillary } \\
\text { choroidal melanoma } \\
\text { overhanging optic disc }\end{array}$ & $\begin{array}{l}\text { Mean } 56 \\
\text { months }\end{array}$ & $\begin{array}{l}15 \text { patients } \\
\text { (13\%) }\end{array}$ & -- & -- & -- \\
\hline $\begin{array}{l}\text { Gunduz et al. } \\
2010 \text { [12] }\end{array}$ & 24 patients & $\begin{array}{l}\text { Choroidal melanoma } \\
\text { treated with ruthenium } \\
\text { brachytherapy alone }\end{array}$ & -- & $\begin{array}{c}2 / 24 \\
(8.3 \%)\end{array}$ & -- & -- & -- \\
\hline $\begin{array}{l}\text { Verschueren } \\
\text { et al. } 2010 \text { [13] }\end{array}$ & 430 patients & $\begin{array}{l}\text { Choroidal } \\
\text { melanoma }\end{array}$ & $\begin{array}{l}\text { Median } 50 \\
\text { months }\end{array}$ & $\begin{array}{c}51 / 430 \\
(11.9 \%)\end{array}$ & $\begin{array}{l}30.4 \text { months } \\
\text { (range } 0 \text { - } 68.4 \\
\text { months) }\end{array}$ & $\begin{array}{c}76.5 \% \text { at } 5 \\
\text { years, } 69.1 \% \\
\text { at } 10 \text { years }\end{array}$ & -- \\
\hline $\begin{array}{l}\text { Karlovits et al. } \\
2011 \text { [14] }\end{array}$ & 35 patients & $\begin{array}{l}\text { Choroidal melanoma } \\
\text { treated with iodine } \\
\text { brachytherapy }\end{array}$ & $\begin{array}{l}\text { Median } 45 \\
\text { months }\end{array}$ & $\begin{array}{c}5 / 35 \\
(14.3 \%)\end{array}$ & $\begin{array}{l}\text { Median } 58 \\
\text { months }\end{array}$ & -- & -- \\
\hline $\begin{array}{l}\text { Newman et al. } \\
2011[15]\end{array}$ & 50 patients & $\begin{array}{l}\text { Subfoveal choroidal } \\
\text { melanomas with } \geq 6 \\
\text { months of follow-up }\end{array}$ & $\begin{array}{l}\text { Median } 54 \\
\text { months }\end{array}$ & $2 / 50(4 \%)$ & -- & -- & -- \\
\hline $\begin{array}{l}\text { Sagoo et al. } \\
2011[22]\end{array}$ & 650 patients & $\begin{array}{l}\text { Juxtapapillary } \\
\text { choroidal melanoma } \\
\text { treated with plaque } \\
\text { brachytherapy }\end{array}$ & -- & $\begin{array}{l}11 \% \text { at } 5 \text { years, } \\
24 \% \text { at } 10 \text { years }\end{array}$ & -- & -- & $\begin{array}{l}\text { High intraocular } \\
\text { pressure, greater } \\
\text { tumor base }\end{array}$ \\
\hline $\begin{array}{l}\text { Chang et al. } \\
2012 \text { [21] }\end{array}$ & 150 patients & $\begin{array}{l}\text { Choroidal melanoma } \\
\text { treated with iodine } \\
\text { brachytherapy }\end{array}$ & $\begin{array}{l}\text { Mean } 21.5 \\
\text { months, } \\
\text { median } 17.3 \\
\text { months }\end{array}$ & $\begin{array}{l}9 / 150 \\
(6.0 \%)\end{array}$ & -- & -- & -- \\
\hline $\begin{array}{l}\text { Finger et al. } \\
2012 \text { [16] }\end{array}$ & 24 patients & $\begin{array}{l}\text { Choroidal melanomas } \\
\text { within } 1.5 \mathrm{~mm} \text { of } \\
\text { optic disc treated with } \\
\text { slotted radiotherapy }\end{array}$ & $\begin{array}{l}\text { Mean } 23 \\
\text { months }\end{array}$ & $\begin{array}{c}0 / 24 \\
(0.0 \%)\end{array}$ & -- & -- & -- \\
\hline $\begin{array}{l}\text { Berry et al. } \\
2013 \text { [17] }\end{array}$ & 82 patients & $\begin{array}{l}\text { Medium sized } \\
\text { choroidal melanomas }\end{array}$ & $\begin{array}{l}\text { Median } 46.8 \\
\text { months }\end{array}$ & $\begin{array}{c}9 / 82 \\
(10.9 \%)\end{array}$ & $\begin{array}{c}41.0 \text { months } \\
\text { (standard } \\
\text { deviation } \\
{[\mathrm{SD}]=34.7} \\
\text { months) }\end{array}$ & $\begin{array}{l}89 \% \text { at } 60 \\
\text { months }\end{array}$ & -- \\
\hline $\begin{array}{l}\text { Caminal et al. } \\
2013 \text { [18] }\end{array}$ & 54 patients & $\begin{array}{l}\text { Choroidal melanoma } \\
\text { treated with iodine } \\
\text { brachytherapy }\end{array}$ & $\begin{array}{l}\text { Mean } 59.47 \\
\text { months }\end{array}$ & $\begin{array}{c}11 / 54 \\
(20.4 \%)\end{array}$ & -- & $\begin{array}{c}84.2 \% \text { at } \\
5 \text { years }\end{array}$ & -- \\
\hline $\begin{array}{l}\text { Semenova et al. } \\
2013 \text { [19] }\end{array}$ & 72 patients & $\begin{array}{l}\text { Small choroidal } \\
\text { melanomas }\end{array}$ & $\begin{array}{l}\text { Mean } 54 \\
\text { months }\end{array}$ & $\begin{array}{c}0 / 72 \\
(0.0 \%)\end{array}$ & -- & -- & -- \\
\hline $\begin{array}{l}\text { Semenova et al. } \\
\quad 2014 \text { [20] }\end{array}$ & 47 patients & $\begin{array}{c}\text { T3 or T4 sized choroidal } \\
\text { melanomas treated } \\
\text { with palladium } \\
\text { brachytherapy }\end{array}$ & $\begin{array}{l}\text { Median } 47 \\
\text { months }\end{array}$ & $\begin{array}{c}15 / 47 \\
(31.9 \%)\end{array}$ & -- & -- & -- \\
\hline
\end{tabular}


Table 14. Distant metastasis outcomes following proton beam therapy for choroidal melanoma.

\begin{tabular}{|c|c|c|c|c|c|c|c|}
\hline Study & Sample Size & Tumor Type & $\begin{array}{l}\text { Duration of } \\
\text { Follow-Up }\end{array}$ & $\begin{array}{c}\text { Number (\%) with } \\
\text { Distant Metastasis } \\
\text { at End of } \\
\text { Follow-Up }\end{array}$ & $\begin{array}{l}\text { Mean Time } \\
\text { to Metastasis }\end{array}$ & $\begin{array}{l}\text { Percentage with } \\
\text { Metastasis-Free } \\
\text { Survival }\end{array}$ & $\begin{array}{c}\text { Predictors of Distant } \\
\text { Metastasis after } \\
\text { Treatment }\end{array}$ \\
\hline $\begin{array}{c}\text { Damato et al. } \\
2005 \text { [23] }\end{array}$ & 349 patients & $\begin{array}{l}\text { Choroidal } \\
\text { melanoma }\end{array}$ & $\begin{array}{c}\text { Median } 3.1 \\
\text { years }\end{array}$ & $\begin{array}{c}2.5 \% \text { died from } \\
\text { metastasis at } 2 \\
\text { years, } 10.0 \% \text { at } \\
5 \text { years, } 14.1 \% \text { at } \\
8 \text { years }\end{array}$ & -- & -- & $\begin{array}{l}\text { Longest basal tumor } \\
\text { dimension, tumor } \\
\text { height, extraocular } \\
\text { tumor extension }\end{array}$ \\
\hline $\begin{array}{c}\text { Conway et al. } \\
2006 \text { [9] }\end{array}$ & 21 patients & $\begin{array}{c}\text { Extra large choroidal } \\
\text { or ciliochoroidal } \\
\text { melanomas }\end{array}$ & $\begin{array}{l}\text { Median } 28 \\
\text { months }\end{array}$ & $3 / 21(14.3 \%)$ & -- & -- & $\begin{array}{l}\text { Characteristics of } \\
\text { patients with metastasis } \\
\text { (no multivariate } \\
\text { modeling performed): } \\
\text { Mean age } 80.7 \text { years, } \\
\text { maximum basal tumor } \\
\text { diameter } 21-24.4 \mathrm{~mm}\end{array}$ \\
\hline $\begin{array}{l}\text { Mosci et al. } \\
2012[24]\end{array}$ & 72 patients & $\begin{array}{c}\text { T3 and T4 choroidal } \\
\text { melanomas }\end{array}$ & $\begin{array}{c}\text { Mean } 53.4 \\
\text { months }\end{array}$ & & -- & $\begin{array}{l}72 \% \text { at } \\
5 \text { years }\end{array}$ & -- \\
\hline $\begin{array}{l}\text { Tran et al. } \\
2012 \text { [25] }\end{array}$ & 59 patients & $\begin{array}{c}\text { Peripapillary choroidal } \\
\text { melanoma }\end{array}$ & $\begin{array}{c}\text { Median } 63 \\
\text { months }\end{array}$ & 15/59 (25.4\%) & $\begin{array}{l}\text { Median } 38 \\
\text { months }\end{array}$ & $\begin{array}{l}82 \% \text { at } \\
5 \text { years }\end{array}$ & -- \\
\hline $\begin{array}{c}\text { Schonfeld } \\
\text { et al. } 2014 \\
\text { [26] }\end{array}$ & 18 patients & $\begin{array}{l}\text { Choroidal melanoma } \\
\text { in intermediate zone } \\
\text { of fundus treated with } \\
\text { proton beam therapy } \\
\text { only }\end{array}$ & $\begin{array}{l}\text { Median } 77.2 \\
\text { months }\end{array}$ & $\begin{array}{c}1 / 18 \text { patients } \\
\text { (5.6\%) died from } \\
\text { metastasis }\end{array}$ & -- & -- & -- \\
\hline
\end{tabular}

\section{Acknowledgements}

This work was generously supported by the George E. and Ruth Moss Trust and an unrestricted grant from the Research to Prevent Blindness.

\section{References}

[1] Singh, A.D. and Topham, A. (2003) Incidence of Uveal Melanoma in the United States: 1973-1997. Ophthalmology, 110, 956-961. http://dx.doi.org/10.1016/S0161-6420(03)00078-2

[2] Inskip, P.D., Devesa, S.S. and Fraumeni Jr., J.F. (2003) Trends in the Incidence of Ocular Melanoma in the United States, 1974-1998. Cancer Causes Control, 14, 251-257. http://dx.doi.org/10.1023/A:1023684502638

[3] Singh, A.D., Bergman, L. and Seregard, S. (2005) Uveal Melanoma: Epidemiological Aspects. Ophthalmology Clinics of North America, 18, 75-84. http://dx.doi.org/10.1016/j.ohc.2004.07.002

[4] Kujala, E., Makitie, T. and Kivela, T. (2003) Very Long-Term Prognosis of Patients with Malignant Uveal Melanoma. Investigative Ophthalmology \& Visual Science, 44, 4651-4469. http://dx.doi.org/10.1167/iovs.03-0538

[5] Damato, B.E., Heimann, H., Kalirai, H. and Coupland, S.E. (2014) Age, Survival Predictors, and Metastatic Death in Patients with Choroidal Melanoma: Tentative Evidence of a Therapeutic Effect on Survival. JAMA Ophthalmology, 132, 605-613. http://dx.doi.org/10.1001/jamaophthalmol.2014.77

[6] Diener-West, M., Earle, J.D., Fine, S.L., et al. (2001) The COMS Randomized Trial of Iodine 125 Brachytherapy for Choroidal Melanoma, III: Initial Mortality Findings. COMS Report No. 18. Archives of Ophthalmology, 119, 969-982. http://dx.doi.org/10.1001/archopht.119.7.969

[7] Wang, Z., Nabhan, M., Schild, S.E., et al. (2013) Charged Particle Radiation Therapy for Uveal Melanoma: A Systematic Review and Meta-Analysis. International Journal of Radiation Oncology*Biology*Physics, 86, 18-26. http://dx.doi.org/10.1016/j.ijrobp.2012.08.026

[8] Wen, J.C., Oliver, S.C. and McCannel, T.A. (2009) Ocular Complications Following I-125 Brachytherapy for Choroidal Melanoma. Eye, 23, 1254-1268. http://dx.doi.org/10.1038/eye.2009.43

[9] Conway, R.M., Poothullil, A.M., Daftari, I.K., et al. (2006) Estimates of Ocular and Visual Retention Following Treatment of Extra-Large Uveal Melanomas by Proton Beam Radiotherapy. Archives of Ophthalmology, 124, 838-843. 
http://dx.doi.org/10.1001/archopht.124.6.838

[10] Sagoo, M.S., Shields, C.L., Mashayekhi, A., et al. (2007) Plaque Radiotherapy for Choroidal Melanoma Encircling the Optic Disc (Circumpapillary Choroidal Melanoma). Archives of Ophthalmology, 125, 1202-1209. http://dx.doi.org/10.1001/archopht.125.9.1202

[11] Sagoo, M.S., Shields, C.L., Mashayekhi, A., Freire, J., Emrich, J., Reiff, J., Komarnicky, L. and Shields, J.A. (2008) Plaque Therapy for Juxtapapillary Choroidal Melanoma Overhanging the Optic Disc in 141 Consecutive Patients. Archives of Ophthalmology, 126, 1515-1522. http://dx.doi.org/10.1001/archopht.126.11.1515

[12] Gunduz, K., Kurt, R.A., Akmese, H.E., Köse, K. and Uçakhan-Gündüz, Ö. (2010) Ruthenium-106 Plaque Radiotherapy Alone or in Combination with Transpupillary Thermotherapy in the Management of Choroidal Melanoma. Japanese Journal of Ophthalmology, 54, 338-343. http://dx.doi.org/10.1007/s10384-010-0821-1

[13] Verschueren, K.M., Creutzberg, C.L., Schalij-Delfos, N.E., Ketelaars, M., Klijsen, F.L.L., Haeseker, B.I., Ligtenberg, S.M.B., Keunen, J.E.E. and Marijnen, C.A.M. (2010) Long-Term Outcomes of Eye-Conserving Treatment with Ruthenium ${ }^{106}$ Brachytherapy for Choroidal Melanoma. Radiotherapy and Oncology, 95, 332-338. http://dx.doi.org/10.1016/j.radonc.2010.03.023

[14] Karlovits, B., Trombetta, M.G., Verstraeten, T., Johnson, M., Wong, M. and Karlovits, S. (2011) Local Control and Visual Acuity Following Treatment of Medium-Sized Ocular Melanoma Using a Contact Eye Plaque: A Single Surgeon Experience. Brachytherapy, 10, 228-231. http://dx.doi.org/10.1016/j.brachy.2010.09.003

[15] Newman, H., Chin, K.J. and Finger, P.T. (2011) Subfoveal Choroidal Melanoma: Pretreatment Characteristics and Response to Plaque Radiation Therapy. Archives of Ophthalmology, 129, 892-898. http://dx.doi.org/10.1001/archophthalmol.2011.161

[16] Finger, P.T., Chin, K.J. and Tena, L.B. (2012) A Five-Year Study of Slotted Eye Plaque Radiation Therapy for Choroidal Melanoma: Near, Touching, or Surrounding the Optic Nerve. Ophthalmology, 119, 415-422.

http://dx.doi.org/10.1016/j.ophtha.2011.08.017

[17] Berry, J.L., Dandapani, S.V., Stevanovic, M., Lee, T.C., Astrahan, M., Murphree, A.L. and Kim, J.W. (2013) Outcomes of Choroidal Melanomas Treated with Eye Physics: A 20-Year Review. JAMA Ophthalmology, 131, 1435-1442. http://dx.doi.org/10.1001/jamaophthalmol.2013.4422

[18] Caminal, J.M., Mejia, K., Masuet-Aumatell, C., Arias, L., Piulats, J.M., Gutierrez, C., Pera, J., Catala, J., Rubio, M. and Arruga, J. (2013) Endoresection versus Iodine-125 Plaque Brachytherapy for the Treatment of Choroidal Melanoma. American Journal of Ophthalmology, 156, 334-342. http://dx.doi.org/10.1016/j.ajo.2013.03.036

[19] Semenova, E. and Finger, P.T. (2013) Palladium-103 Radiation Therapy for Small Choroidal Melanoma. Ophthalmology, 120, 2353-2357. http://dx.doi.org/10.1016/j.ophtha.2013.04.017

[20] Semenova, E. and Finger, P.T. (2014) Palladium-103 Plaque Radiation Therapy for American Joint Committee on Cancer T3- and T4-Staged Choroidal Melanomas. JAMA Ophthalmology, 132, 205-213. http://dx.doi.org/10.1001/jamaophthalmol.2013.5677

[21] Chang, M.Y., Kamrava, M., Demanes, J., Agazaryan, N., Lamb, J., Moral, J.N., Almanzor, R. and McCannel, T.A. (2012) Intraoperative Ultrasonography-Guided Positioning of Iodine-125 Plaque Brachytherapy in the Treatment of Choroidal Melanoma. Ophthalmology, 119, 1073-1077. http://dx.doi.org/10.1016/j.ophtha.2011.11.011

[22] Sagoo, M.S., Shields, C.L., Mashayekhi, A., Freire, J., Emrich, J., Reiff, J., Komarnicky, L. and Shields, J.A. (2011) Plaque Radiotherapy for Juxtapapillary Choroidal Melanoma. Ophthalmology, 118, 402-407. http://dx.doi.org/10.1016/j.ophtha.2010.06.007

[23] Damato, B., Kacperek, A., Chopra, M., Campbell, I.R. and Errington, R.D. (2005) Proton Beam Radiotherapy of Choroidal Melanoma: The Liverpool-Clatterbridge Experience. International Journal of Radiation Oncology, Biology, Physics, 62, 1405-1411. http://dx.doi.org/10.1016/j.ijrobp.2005.01.016

[24] Mosci, C., Lanza, F.B., Barla, A., Mosci, S., Hérault, J., Anselmi, L. and Truini, M. (2012) Comparison of Clinical Outcomes for Patients with Large Choroidal Melanoma after Primary Treatment with Enucleation or Proton Beam Radiotherapy. Ophthalmologica, 227, 190-196. http://dx.doi.org/10.1159/000334401

[25] Tran, E., Ma, R., Paton, K., Blackmore, E. and Pickles, T. (2012) Outcomes of Proton Radiation Therapy for Peripapillary Choroidal Melanoma at the BC Cancer Agency. International Journal of Radiation Oncology, Biology, Physics, 83, 1425-1431. http://dx.doi.org/10.1016/j.ijrobp.2011.10.017

[26] Schonfeld, S., Cordini, D., Riechardt, A.I., Seibel, I., Willerding, G., Bechrakis, N.E., Moser, L. and Joussen, A.M. (2014) Proton Beam Therapy Leads to Excellent Local Control Rates in Choroidal Melanoma in the Intermediate Fundus Zone. American Journal of Ophthalmology, 158, 1184-1191. http://dx.doi.org/10.1016/j.ajo.2014.08.016

[27] Cook, D.J., Mulrow, C.D. and Haynes, R.B. (1997) Systematic Reviews: Synthesis of Best Evidence for Clinical Decisions. Annals of Internal Medicine, 126, 376-380. http://dx.doi.org/10.7326/0003-4819-126-5-199703010-00006

[28] US Library of Medicine, National Institutes of Health. PubMed.gov. Accessed 8 December 2015. 
http://www.ncbi.nlm.nih.gov/pubmed

[29] Bergman, L., Nilsson, B., Lundell, G., Lundell, M. and Seregard, S. (2005) Ruthenium Brachytherapy for Uveal Melanoma, 1979-2003: Survival and Functional Outcomes in the Swedish Population. Ophthalmology, 112, 834-840. http://dx.doi.org/10.1016/j.ophtha.2004.11.038

[30] Finger, P.T., Chin, K.J. and Yu, G.P., Palladium-103 for Choroidal Melanoma Study Group (2010) Risk Factors for Radiation Maculopathy after Ophthalmic Plaque Radiation for Choroidal Melanoma. American Journal of Ophthalmology, 149, 608-615. http://dx.doi.org/10.1016/j.ajo.2009.11.006

[31] Marconi, D.G., de Castro, D.G., Reboucas, L.M., Gil, G.O.B., Fogaroli, R.C., Maia, M.A.C., Silva, M.L.G., Pellizzon, A.C.A. and Chojniak, M.M.M. (2013) Tumor Control, Eye Preservation, and Visual Outcomes of Ruthenium Plaque Brachytherapy for Choroidal Melanoma. Brachytherapy, 12, 235-239. http://dx.doi.org/10.1016/j.brachy.2012.01.012

[32] Sagoo, M.S., Shields, C.L., Emrich, J., Mashayekhi, A., Komarnicky, L. and Shields, J.A. (2014) Plaque Radiotherapy for Juxtapapillary Choroidal Melanoma: Treatment Complications and Visual Outcomes in 650 Consecutive Cases. JAMA Ophthalmology, 132, 697-702. http://dx.doi.org/10.1001/jamaophthalmol.2014.111

[33] Kim, I.K., Lane, A.M., Egan, K.M., Munzenrider, J. and Gragoudas, E.S. (2010) Natural History of Radiation Papillopathy after Proton Beam Irradiation of Parapapillary Melanoma. Ophthalmology, 117, 1617-1622. http://dx.doi.org/10.1016/j.ophtha.2009.12.015

[34] Riechardt, A.I., Cordini, D., Willerding, G.D., Georgieva, I., Weber, A., Seibel, I., et al. (2014) Proton Beam Therapy of Parapapillary Choroidal Melanoma. American Journal of Ophthalmology, 157, 1258-1265. http://dx.doi.org/10.1016/j.ajo.2014.02.032

[35] Jampol, L.M., Moy, C.S., Murray, T.G., Reynolds, S.M., Albert, D.M., Schachat, A.P., et al. (2002) The COMS Randomized Trial of Iodine 125 Brachytherapy for Choroidal Melanoma: IV. Local Treatment Failure and Enucleation in the First 5 Years after Brachytherapy. COMS Report No. 19. Ophthalmology, 109, 2197-2206. http://dx.doi.org/10.1016/S0161-6420(02)01277-0

[36] Brandberg, Y., Kock, E., Oskar, K., Trampe, E.A. and Seregard, S. (2000) Psychological Reactions and Quality of Life in Patients with Posterior Uveal Melanoma Treated with Ruthenium Plaque Therapy or Enucleation: A One Year Follow-Up Study. Eye, 14, 839-846. http://dx.doi.org/10.1038/eye.2000.233

[37] Macdonald, E.C., Cauchi, P. and Kemp, E.G. (2011) Proton Beam Therapy for the Treatment of Uveal Melanoma in Scotland. British Journal of Ophthalmology, 95, 1691-1695. http://dx.doi.org/10.1136/bjo.2010.195594

[38] Levin, L.A., Gragoudas, E.S. and Lessell, S. (2000) Endothelial Cell Loss in Irradiated Optic Nerves. Ophthalmology, 107, 370-374. http://dx.doi.org/10.1016/S0161-6420(99)00054-8

[39] Kline, L.B., Kim, J.Y. and Ceballos, R. (1985) Radiation Optic Neuropathy. Ophthalmology, 92, 1118-1126. http://dx.doi.org/10.1016/S0161-6420(85)33898-8

[40] Krema, H., Heydarian, M., Beiki-Ardakani, A., Weisbrod, D., Xu, W., Laperriere, N.J. and Sahgal, A. (2013) Dosimetric and Late Radiation Toxicity Comparison between Iodine-125 Brachytherapy and Stereotactic Radiation Therapy for Juxtapapillary Choroidal Melanoma. International Journal of Radiation Oncology, Biology, Physics, 86, 510-515. http://dx.doi.org/10.1016/j.ijrobp.2013.02.005

[41] Archer, D.B., Amoaku, W.M. and Gardiner, T.A. (1991) Radiation Retinopathy-Clinical, Histopathological, Ultrastructural and Experimental Correlations. Eye, 5, 239-251. http://dx.doi.org/10.1038/eye.1991.39

[42] Amoaku, W.M. and Archer, D.B. (1990) Fluorescein Angiographic Features, Natural Course and Treatment of Radiation Retinopathy. Eye, 4, 657-667. http://dx.doi.org/10.1038/eye.1990.93

[43] Correa, R., Pera, J., Gomez, J., Polo, A., Gutiérrez, C., Caminal, J.M., Modolell, I., Navarro, V. and Guedea, F. (2009) ${ }^{125}$ I Episcleral Plaque Brachytherapy in the Treatment of Choroidal Melanoma: A Single-Institution Experience in Spain. Brachytherapy, 8, 290-296. http://dx.doi.org/10.1016/j.brachy.2009.03.189

[44] Bianciotto, C., Shields, C.L., Pirondini, C., Mashayekhi, A., Furuta, M. and Shields, J.A. (2010) Proliferative Radiation Retinopathy after Plaque Radiotherapy for Uveal Melanoma. Ophthalmology, 117, 1005-1012. http://dx.doi.org/10.1016/j.ophtha.2009.10.015

[45] Parsons, J.T., Fitzgerald, C.R., Hood, C.I., Ellingwood, K.E., Bova, F.J. and Million, R.R. (1983) The Effects of Irradiation on the Eye and Optic Nerve. International Journal of Radiation Oncology, Biology, Physics, 9, 609-622. http://dx.doi.org/10.1016/0360-3016(83)90225-0

[46] Collaborative Ocular Melanoma Study Group (2007) Incidence of Cataract and Outcomes after Cataract Surgery in the First 5 Years after Iodine 125 Brachytherapy in the Collaborative Ocular Melanoma Study: COMS Report No. 27. Ophthalmology, 114, 1363-1371. http://dx.doi.org/10.1016/j.ophtha.2006.10.039

[47] Finger, P.T., Chin, K.J., Yu, G.P. and Patel, N.S. (2011) Risk Factors for Cataract after Palladium-103 Ophthalmic Plaque Radiation Therapy. International Journal of Radiation Oncology, Biology, Physics, 80, 800-806. http://dx.doi.org/10.1016/j.ijrobp.2010.02.051 
[48] Aoyama, T., Mastrangelo, M.J., Berd, D., Nathan, F.E., Shields, C.L., Shields, J.A., Rosato, E.L., Rosato, F.E. and Sato, T. (2000) Protracted Survival after Resection of Metastatic Uveal Melanoma. Cancer, 89, 1561-1568. http://dx.doi.org/10.1002/1097-0142(20001001)89:7<1561::AID-CNCR21>3.0.CO;2-R

[49] Roland, K., Hayungs, J., Bronfeld, N., Sauerwein, W., Höffken, K. and Seeber, S. (1993) Prognosis and Treatment of Disseminated Uveal Melanoma. Cancer, 72, 2219-2223. http://dx.doi.org/10.1002/1097-0142(19931001)72:7<2219::AID-CNCR2820720725>3.0.CO;2-J 